\title{
Extended dual antiplatelet therapy after acute myocardial infarction. Current evidence and future perspectives
}

\author{
Nicola Cosentino ${ }^{1}$, Jeness Campodonico ${ }^{1}$, Valentina Milazzo ${ }^{1}$, Katia Celentano ${ }^{1}$, Marco Moltrasio ${ }^{1}$, \\ Pompilio Faggiano ${ }^{2}$, Giancarlo Marenzi ${ }^{1}$ \\ ${ }^{1}$ Centro Cardiologico Monzino IRCCS, Milan; ${ }^{2}$ Cardiology Unit, Spedali Civili, University of Brescia, Italy
}

\begin{abstract}
Patients with acute myocardial infarction (AMI) are at increased risk of recurrent ischemic events after hospital discharge, despite optimal medical therapy. Current practice guidelines strongly encourage the early assessment of the residual ischemic risk in post-AMI patients, in order to identify those who may benefit from a prolonged dual antiplatelet therapy. To this end, some scoring systems have been proposed. However, most scores were developed for patients with stable coronary artery disease undergoing percutaneous coronary intervention. Moreover, nearly all failed to be implemented in everyday clinical practice, probably because of the perceived complexity due to the large number of incorporated variables. Therefore, the identification of the ideal AMI patient who can benefit from a prolonged (beyond 1 year after the index event) dual antiplatelet therapy remains to be clarified, especially when the bleeding risk associated with such therapy is considered. In this review, we summarize the current evidence on the prolonged use of dual antiplatelet therapy after AMI, with a special focus on recent advances regarding the identification of high-risk patients who may derive a favorable net clinical benefit from such a therapeutic strategy.
\end{abstract}

\footnotetext{
Correspondence: Giancarlo Marenzi, Centro Cardiologico Monzino, Via Parea 4, 20138 Milan, Italy.

Tel. +39.02.580021 - Fax: +39.02.58002287

E-mail: giancarlo.marenzi@ccfm.it
}

Key words: Acute myocardial infarction; dual antiplatelet therapy; risk score; ischemic risk; bleeding.

Received for publication: 12 February 2019.

Accepted for publication: 6 May 2019.

CCopyright N. Cosentino et al., 2019

Licensee PAGEPress, Italy

Monaldi Archives for Chest Disease 2019; 89:1046

doi: 10.4081/monaldi.2019.1046

This article is distributed under the terms of the Creative Commons Attribution Noncommercial License (by-nc 4.0) which permits any noncommercial use, distribution, and reproduction in any medium, provided the original author(s) and source are credited.

\section{Introduction}

Patients with acute myocardial infarction (AMI) are at increased risk of recurrent ischemic events after hospital discharge, despite current optimal medical therapy [1-3], with each episode associated with an increase in mortality [4-7]. In particular, the risk of recurrent AMI is greatest during the first 30 days and remains significantly elevated in the first year, with reported 1 -year rates that dropped from about $25 \%$ [8] in the early nineties to the current $5-7 \%[2,9,10]$.

This heightened predisposition to athero-thrombotic events has been attributed to a persistent elevated platelet activation for a considerable period after AMI [11,12], suggesting that prolonged inhibition of platelet function may be needed to reduce the risk of recurrent ischemic events. Accordingly, the benefits of dual antiplatelet therapy (APT) was first established by the Clopidogrel in Unstable Angina to Prevent Recurrent Events (CURE) [13], ClOpidogrel and Metoprolol in Myocardial Infarction Trial/Second Chinese Cardiac Study (COMMITT/CCS-2) [14], and CLopiodogrel as Adjunctive ReperfusIon TherapY (CLARITY)-Thrombolysis In Myocardial Infarction (TIMI) 28 [15] trials. Combined aspirin and clopidogrel therapy reduced the 1-year incidence of cardiovascular events by approximately $20 \%$, compared with aspirin alone. More potent P2Y12 receptor inhibition with either prasugrel or ticagrelor was superior to clopidogrel in the subsequent TRial to assess Improvement in Therapeutic Outcomes by optimizing platelet InhibitioN with prasugrel Thrombolysis In Myocardial Infarction 38 (TRITON-TIMI 38) and Study of Platelet Inhibition and Patient Outcomes (PLATO) trials [16,17]. However, a higher predisposition to new ischemic events may persist for years following an AMI, as clearly demonstrated in recent registries and subgroup analyses of prior trials [1-7]. As such, these patients may have a persistent patho-biology, which would justify the extension of dual APT beyond the first year after the index event. Nevertheless, dual APT is strongly recommended for only up to one year for reduction of cardiovascular events in patients with a prior AMI, with a weak (IIb) recommendation to continue thereafter $[18,19]$.

Recently, two large randomized trials demonstrated that extended duration of dual APT significantly reduced atherothrombotic events in patients one year or more after AMI at the expense of higher bleeding [20,21]. Given these findings and the heterogeneity in results of other trials and meta-analyses testing prolonged dual APT duration [20-24], the net clinical benefit of dual APT beyond one year for secondary prevention in patients with AMI is still controversial.

The objective of the present review is to summarize the current evidences supporting dual APT extension beyond one year 
after AMI, with a special focus on the identification of potential candidates who may benefit the most from such a therapeutic strategy.

\section{Long-term residual ischemic and bleeding risk after AMI}

While much information characterizes the short-term and medium-term prognosis of AMI patients, long-term ( $>1$ year) postAMI outcome has received less extensive attention. Only recently, registries and randomized controlled trials have addressed this issue $[1-7,13-15,18,19]$.

In a large Swedish registry study, which included 97,254 patients discharged after AMI, the risk of non-fatal AMI, non-fatal stroke, or cardiovascular death (primary composite endpoint) during the first year after the index event was $18.3 \%$ [2]. Although the risk was lower in the subsequent three years, it remained relatively high with about $20 \%$ of patients experiencing the primary endpoint during the following three years [2]. More recently, a further large four-country analysis, including more than 100,000 survivors of AMI aged $\geq 65$ years, showed an annual risk of death one year onwards after AMI that was more than double that of a similar general population, with about half of deaths due to cardiovascular disease [25]. Indeed, death, stroke, or further AMI after the first year occurred in about one-third of patients during the subsequent three years. However, in this study [25], the primary composite end-point included all-cause death, that reached $36 \%$ for the United States population, that had a higher rate of comorbidities, such as chronic obstructive pulmonary disease, renal failure, and history of more than 1 AMI or heart failure, factors that may be linked to non athero-thrombotic causes of death. Similarly, in the Harmonizing Outcomes with Revascularization and Stents in Acute Myocardial Infarction (HORIZONS-AMI) trial, a prospective study of patients with ST-elevation myocardial infarction (STEMI) who were all treated with primary percutaneous coronary intervention (PCI), the 3-year incidence of recurrent AMI was $6.9 \%[26]$.

Finally, data from post-AMI populations derived from the recent Dual Antiplatelet Therapy trial (DAPT) and the Prevention of Cardiovascular Events in Patients with Prior Heart Attack Using Ticagrelor Compared to Placebo on a Background of AspirinThrombolysis in Myocardial Infarction 54 (PEGASUS-TIMI 54) trial revealed annual rates of major adverse cardiac events in patients treated with aspirin only of $4 \%$ and $3 \%$ for the same outcome, an expected lower figure as compared to that reported in real-world populations [20,21]. Of note, in the PEGASUS-TIMI 54 trial, the risk of ischemic events continued for several years without evidence of decreasing risk more than five years from the qualifying AMI [27].

Not only recurrent ischemic events are likely after AMI but also bleeding events may occur in patients following AMI treated with anti-thrombotic agents. Indeed, a detrimental liaison exists between ischemic recurrences, bleeding and mortality. Registries and trials have consistently shown that major bleeding is associated with an increase in mortality, which could partially thwart the benefits of dual APT [28-31]. Several mechanisms have been proposed to explain the link between bleeding and mortality, including rebound hypercoagulability and ischemic events due to discontinuation of antithrombotic treatments [32]. Although bleeding may be a life-threatening complication of dual APT, it should be underlined that the incidence of major bleeding events reported in con- trolled trials and registries in AMI patients treated with dual APT is low, especially when compared to its ischemic counterpart. Indeed, most studies reported a rate of fatal bleeding within the first year after AMI always below 1\% [16,17,29]. Moreover, in a study population including 14,963 patients derived from 8 multicenter randomized clinical trials, undergoing coronary stent implantation and treated with dual APT, out-of-hospital major or minor bleeding occurred with a yearly incidence of $1.2 \%$ at a median follow-up of about two years (with half of bleeding events being major) [33].

A similar figure was reported in a recent real-world AMI cohort, in which major bleeding rate (fatal, bleeding requiring transfusion, and hemorrhagic stroke) during the first year after the index event was very low (about $0.2 \%$ ) [34]. Moreover, evidences now showed that in patients who have tolerated dual APT for one year, without experiencing a bleeding event, long-term tolerability, and hence compliance with therapy, is very high, with annual rates of drug discontinuation similar to those seen with placebo [35]. Notably, the strategy of continuing dual APT uninterrupted beyond twelve months from AMI offered a greater magnitude of benefit (27\% relative risk reduction) than reinitiating in patients with a more remote AMI who were event-free while receiving aspirin monotherapy [36].

Therefore, post-AMI patients appear to have a sustained and high risk of recurrent athero-thrombosis and subsequent death. This suggests that, in these patients, surveillance is required beyond twelve months after the index event, in order to update continuously their ischemic and bleeding profile.

\section{Long-term dual apt for secondary prevention of AMI}

As patients with AMI are at higher risk of ischemic recurrences than patients who have coronary artery disease without prior AMI, several trials and subgroup analyses investigated whether a more intensive long-term dual APT in AMI patients is associated with a greater clinical benefit. The Clopidogrel and Aspirin Versus Aspirin Alone for the Prevention of Atherothrombotic Events trial (CHARISMA) [37] included coronary artery disease patients with and without prior AMI who were randomized to clopidogrel plus aspirin or aspirin alone and were treated for a median of 28 months. Although the overall results of the study were neutral, patients with prior AMI had a significant $23 \%$ relative risk reduction in major adverse cardiac events with the addition of clopidogrel to aspirin. In contrast, those without prior AMI showed a trend toward harm. Similarly, in the DAPT study [38], the subgroup of patients with a prior AMI derived a larger ischemic benefit from prolonged treatment with clopidogrel or prasugrel in addition to aspirin (44\% relative risk reduction vs $17 \%$ in patients without AMI). The PEGASUS-TIMI 54 trial demonstrated that the addition of ticagrelor to low-dose aspirin reduces long-term ischemic risk in patients with a history of AMI (1-3 years earlier), with more than 50 years of age, and at least one additional high-risk feature among age $\geq 65$ years, diabetes mellitus, prior AMI, multi-vessel coronary artery disease, and estimated glomerular filtration rate $<60 \mathrm{ml} / \mathrm{min} / 1.73 \mathrm{~m}^{2}$ [21]. Thus, the hypothesis that long-term dual APT reduces ischemic events in patients with prior AMI has been demonstrated with clopidogrel in the CHARISMA trial, clopidogrel and prasugrel in the DAPT trial, and ticagrelor in the PEGASUS-TIMI 54. However, these trials consistently showed that the reduction in the long-term ischemic risk with dual APT is associ- 
ated with increased bleeding risk, albeit generally not of fatal or intracranial bleeding. More recently, in the predicting bleeding complications in patients undergoing stent implantation and subsequent dual antiplatelet therapy (PRECISE-DAPT) analysis [33], considering 14,963 patients (nearly $30 \%$ of whom presented with AMI) treated with dual APT, largely consisting of aspirin and clopidogrel, a longer dual APT duration significantly increased bleeding in patients at high risk, but not in those with lower risk profiles, and exerted a significant ischemic benefit only in this latter group.

Two meta-analyses also examined whether the duration of dual APT in patients who have undergone PCI using drug eluting stent for any indication has an effect on a range of outcomes, including stent thrombosis, cardiovascular death, all-cause mortality, and bleeding [22,23]. Both studies included the same ten trials that compared standard ( $\leq 1$ year) or extended ( $>1$ year) courses of dual APT and concluded that fewer than twelve months of dual APT is associated with significantly lower all-cause mortality compared with greater than twelve months. Moreover, a significantly lower risk of major bleeding and a higher risk of AMI and stent thrombosis were observed in the shorter dual APT groups. However, only a minority of study patients included in the meta-analyses had a history of prior AMI, ranging from $1.6 \%$ to $35 \%$ of patients. It is therefore difficult to extrapolate the results of this overall analysis to patients with AMI specifically. As such, Udell et al. [24] focused their investigation on patients with prior AMI only. In their metaanalysis including $>33,000$ AMI patients they found that extended dual APT (beyond one year) resulted in a $22 \%$ relative risk reduction for major adverse cardiovascular events over a mean followup of about three years. The pooled data in this meta-analysis showed for the first time that there is a significant $15 \%$ reduction in cardiovascular death in post-AMI patients receiving long-term dual APT, with a trend towards reduced all-cause mortality. On the other hand, there was a $27 \%$ relative increase in the risk of major bleeding (from $1.1 \%$ to $1.8 \%$ ), but without significant excess of intracranial or fatal bleeding.

Taken together, these findings support prior research suggesting that the mechanism of long-term cardiovascular benefit with prolonged dual APT in AMI patients is likely an extension of the benefits seen following early treatment, and that anti-thrombotic therapy in AMI patients should be differentiated from that of patients undergoing elective PCI. Notably, the Providing Regional Observations to Study Predictors of Events in the Coronary Tree (PROSPECT) study demonstrated, in acute coronary syndrome patients undergoing PCI, that major adverse cardiovascular events (death from cardiac causes, cardiac arrest, myocardial infarction, or rehospitalization due to unstable or progressive angina) occurring at three-year follow-up were equally attributable to recurrence at the site of culprit lesions and to non-culprit lesions [39]. This, further highlights the critical need to protect the vulnerable patient rather than to prevent ischemic recurrences related to stent only.

\section{Future perspectives}

Current practice guidelines strongly encourage the early assessment of ischemic risk in post-AMI patients, in order to identify those who may benefit from a prolonged dual APT [18,19]. Accordingly, several scoring systems have been proposed to aid clinicians in this decision making [40-46] (Table 1). However, the complexity of some of these risk scores, due to the many variables included, makes their clinical application impractical [40-44]. Moreover, the majority of them were developed for patients under-

Table 1. Most recently proposed scores for ischemic risk assessment in patients with coronary artery disease.

\begin{tabular}{llll}
\hline DAPT [45] & PARIS [46] & CHINA PEACE [44] & Registry \\
Study design & RCT & Registry & 2018 \\
Year of publication & 2016 & 2016 & 3170 \\
Patients in the derivation cohort (n) & 11,648 & 4190 & 1057 \\
Patients in the validation cohort (n) & 8709 & 8130 & $100 \%$ \\
Percentage of AMI patients & $21 \%$ & $25 \%$ & 12 months after AMI \\
Length of follow-up & from 12 to 30 months after PCI & 24 months after PCI & 0.73 \\
AUC (derivation cohort) & 0.68 & 0.68 & 0.77 \\
AUC (validation cohort) & 0.62 & 0.65 & Age \\
Variables included & Age & ACS & Prior AMI \\
& Smoking & Smoking & Education \\
& Diabetes mellitus & Diabetes mellitus & Prior VTNF \\
& AMI at presentation & ClCr $<60$ ml/min & Hypertension \\
& Prior PCI/AMI & Prior PCI & Angina \\
& Paclitataxel-eluting stent & Prior CABG & Prearrival medical assistance \\
& Stent diameter $<3$ mm & & $>4$ h from symptoms onset \\
& CHF/LEF $<30 \%$ & & LVEF \\
& Vein graft PCI & & Renal dysfunction
\end{tabular}

DAPT, dual antiplatelet therapy trial; PARIS, patterns of non-adherence to antiplatelet regimen in stented patients; CHINA PEACE, China patients-centered evaluative assessment of cardiac events prospective study of AMI; RCT, randomized controlled trial; AMI, acute myocardial infarction; PCI, percutaneous coronary intervention; AUC, area under the curve; ACS, cute coronary syndrome; CICr, creatinine clearance; VF, ventricular fibrillation; $\mathrm{VT}$, ventricular tachycardia; $\mathrm{CABG}$, coronary artery bypass graft; $\mathrm{CHF}$, chronic heart failure; LVEF, left ventricular ejection fraction. 
going PCI, including mostly elective procedures $[41,45,46]$. For instance, the DAPT score was developed, by using data from the DAPT trial, to determine the net clinical benefit of extending dual APT from twelve to thirty months after coronary stenting in 9961 patients, $74 \%$ of whom underwent elective PCI [45]. Thirty-seven candidate variables were considered with eight included in the final model. In the validation cohort, the $c$-statistic was 0.64 for ischemia and 0.64 for bleeding. The Patterns of Non-Adherence to AntiPlatelet Regimen in Stented Patients (PARIS) score, derived from a prospective observational registry, had a model discrimination for ischemia ( $c$-statistic 0.65 ) and bleeding ( $c$-statistic 0.64 ) events comparable to that of the DAPT score [46]. These scores certainly represent a step forward for identification of patients at high-risk for ischemic recurrences after coronary stent implantation. However, some aspects of the studies from which these scores were derived, do not make them fully suitable for the real-world AMI patient. They include patient selection criteria, the predominant dual APT used in trials (mainly aspirin and clopidogrel), and the lack of a specific focus on AMI patients, regardless of PCI. Furthermore, the reproducibility of these scores in different countries remains unclear. Indeed, it was recently reported a poor discrimination for ischemic risk of the DAPT score (c-statistic 0.54) when applied to a large population of Swedish patients, suggesting that risk scores might not be generalizable to all real-world populations [47].

The PEGASUS-TIMI 54 trial was the first study that prospectively addressed the issue of prolonged dual APT in AMI patients only. However, when the PEGASUS-TIMI 54 inclusion criteria are strictly applied to everyday clinical practice, more than $80 \%$ of all AMI patients result to be at high ischemic risk, and should be considered eligible for a prolonged dual APT $[48,49]$. Thus, a further risk stratification should be made in order to identify those AMI patients who may benefit the most from such a therapeutic strategy. To this regard, we recently developed and validated a risk score, ranging from zero to thirteen (Table 2), for ischemic risk prediction in AMI patients, based on the PEGASUS-TIMI 54 criteria [34]. Our score showed a good discrimination accuracy in two independent AMI cohorts ( $c$-statistics 0.70 and 0.68 , respectively). Of note, patients with a score above the identified cut-off value $(\geq 6)$ had an absolute increase in 1-year death and non-fatal AMI risk that was 3-4 times higher than that of patients with a score below this threshold. Moreover, in our validation cohort, ischemic events went on occurring for several years after the qualifying AMI, without any signal of risk reduction (Figure 1). Again, this was particularly true in high-risk patients. Indeed, almost $50 \%$ of patients with a score above the cut-off experienced death or recurrent non-fatal AMI at a median 4-year follow-up. These findings

Table 2. A new risk score for ischemic risk prediction in AMI patients by using the PEGASUS-TIMI 54 criteria. Adapted from Cosentino et al. [34] Int J Cardiol 2019;278:1-6, with permission.

\begin{tabular}{lc} 
& Points \\
Age $>65$ years & 2 \\
eGFR $<60 \mathrm{ml} / \mathrm{min} / 1.73 \mathrm{~m}^{2}$ & 2 \\
\hline Prior AMI & 4 \\
Multi-vessel CAD & 3 \\
\hline Diabetes mellitus & 2 \\
Maximum score & 13 \\
\hline AML acute myocardial infarction; CAD, coronary artery disease; eGFR, estimated glomerular filtration rate.
\end{tabular}

were expected, as they confirm the relevance of risk factors considered in the PEGASUS-TIMI 54 trial and known to be associated with recurrent ischemic events, and they emphasize their independent and synergistic prognostic value [1-7]. In agreement with our results, among 1,592 consecutive patients with acute coronary syndromes treated with PCI and enrolled in the Responsiveness to Clopidogrel and Stent Thrombosis 2-ACS (RECLOSE 2-ACS) study [48] the odds ratios associated to each PEGASUS-TIMI 54 criterion for adverse clinical events occurring one to four years after the index event were similar and around two. Moreover, the ischemic risk progressively rose in parallel with the increase of risk factor number, with each additional variable being associated with a $66 \%$ relative risk increase at long-term follow-up. Thus, although the potential clinical benefit of a prolonged dual APT was not tested in our study population, such therapy may be particularly attractive in AMI patients with heightened ischemic risk that, based on our simple score, can be easily identified before hospital discharge.

Since ischemic and bleeding events share similar risk factors, the benefit on ischemic recurrences of prolonged dual APT after AMI should be carefully balanced against its coupled bleeding risk. In patients at high ischemic risk, a greater absolute risk reduction in cardiovascular events, when compared to the bleeding risk increase, associated with prolonged dual APT may be anticipated. Indeed, in our study, major bleeding event rates during the first year after AMI, when most patients were treated with dual APT, were very low and similar in high- and low-risk groups, supporting a net clinical benefit in patients at high-risk for ischemic recurrences. Interestingly, in the PEGASUS-TIMI 54 trial, extending dual APT for eighteen to thirtysix months beyond the recommended duration of one year yielded a $1-3 \%$ decrease in ischemic events (spontaneous AMI, stent thrombosis, or stroke) at the cost of a $1 \%$ increase in bleeding events among AMI patients [18]. However, this apparent neutral net clinical benefit associated with dual APT should not be misinterpreted. For example, by applying a similar hypothetical ischemic advantage and bleeding drawback to our study population [34], a 30\% relative reduction in ischemic events in the first year of therapy and an equal $30 \%$ increase in major bleeding events would result in an absolute

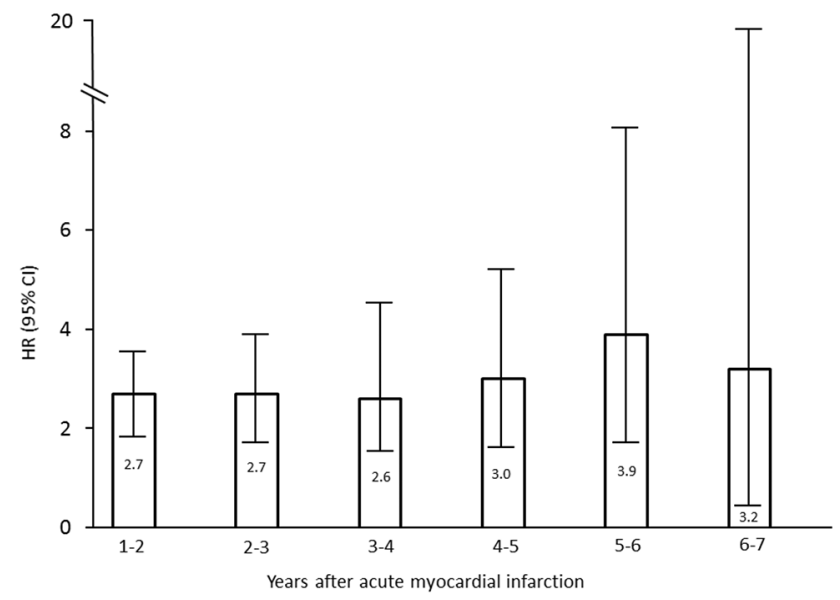

Figure 1. Hazard ratios (HR) and 95\% confidence intervals (CI) for mortality and recurrent non-fatal acute myocardial infarction associated with a risk score $\geq 6$ at every following year after the index event. The risk score refers to the study by Cosentino $e t a l$. [34] Int J Cardiol 2019;278:1-6, with permission. 
reduction of 45 ischemic events (from 151 to 106) and an increase of only 1 (from 3 to 4 ) in major bleeding events (number needed to treat 48 patients and number needed to harm 2170 patients). Therefore, when the effects of a therapy are considered in terms of absolute rather than relative numbers, the net clinical impact can be better appreciated.

Recently, there has been a growing interest in prolonged therapy with novel anticoagulant agents in conjunction with APT to improve outcomes in post-AMI patients. Again, the inherent benefit from anticoagulant therapy has to be weighed against the increased risk of bleeding, and achieving a suitable balance is critical in clinical practice. Some of the novel oral anticoagulants have been assessed for secondary prevention after AMI, with promising results [50,51]. Whether long-term anticoagulation with these drugs, at standard or at lower dose, added to a single or dual APT may represent a potential adjunctive treatment strategy in AMI patients, with or without atrial fibrillation, warrants further investigation, especially in those at heightened ischemic risk.

\section{Conclusions}

Despite great advances in secondary prevention over the last decades, patients retain a high risk of recurrent ischemic events after AMI. Accumulating evidence shows that increased risk likely results from a combination of individual patient characteristics that can be early identified and incorporated into scoring systems. This offers an opportunity to improve outcomes by using a personalized, targeted approach, particularly in terms of dual APT duration. As long as no firm conclusion on the optimal duration of dual APT is drawn, physicians must integrate study results, guideline recommendations, clinical judgment, and continuous patient assessment in choosing the best strategy for each patient.

\section{References}

1. Fox KA, Carruthers KF, Dunbar DR, et al. Underestimated and under-recognized: the late consequences of acute coronary syndrome (GRACE UK-Belgian Study). Eur Heart J 2010;31:2755-64.

2. Jernberg T, Hasvold P, Henriksson M, et al. Cardiovascular risk in post-myocardial infarction patients: nationwide real world data demonstrate the importance of a long-term perspective. Eur Heart J 2015;36:1163-70.

3. Alnasser SM, Huang W, Gore JM, et al. Late consequences of acute coronary syndromes: global registry of acute coronary events (GRACE) follow-up. Am J Med 2015;128:766-75.

4. Mozaffarian D, Benjamin EJ, Go AS, et al. Heart disease and stroke statistics-2016 update: a report from the American Heart Association. Circulation 2016;133:e38-48.

5. Kernis SJ, Harjai KJ, Stone GW, et al. The incidence, predictors, and outcomes of early re infarction after primary angioplasty for acute myocardial infarction. J Am Coll Cardiol 2003;42:1173-7.

6. Hudson MP, Granger CB, Topol EJ, et al. Early re-infarction after fibrinolysis: experience from the global utilization of streptokinase and tissue plasminogen activator (alteplase) for occluded coronary arteries (GUSTO I) and global use of strategies to open occluded coronary arteries (GUSTO III) trials. Circulation 2001;104:1229-35.
7. Kornowski R, Goldbourt U, Zion M, et al. Predictors and longterm prognostic significance of recurrent infarction in the year after a first myocardial infarction. Am J Cardiol 1993;72:883-8.

8. Figueras J, Missorici M, Lidón RM, et al. Coincidental annual distribution of first and second acute myocardial infarction. Am J Cardiol 2002;89:1416-20.

9. Ndrepepa G, Tiroch K, Fusaro M, et al. 5-year prognostic value of no-reflow phenomenon after percutaneous coronary intervention in patients with acute myocardial infarction. J Am Coll Cardiol 2010;55:2383-9.

10. Smolina K, Wright FL, Rayner M, Goldacre MJ. Long-term survival and recurrence after acute myocardial infarction in England, 2004 to 2010. Circ Cardiovasc Qual Outcomes 2012;5:532-40.

11. Trip MD, Cats VM, van Capelle FJ, Vreeken J. Platelet hyperreactivity and prognosis in survivors of myocardial infarction. N Engl J Med 1990;322:1549-54.

12. Lina Badimon, Teresa Padró, Gemma Vilahur. Atherosclerosis, platelets and thrombosis in acute ischaemic heart disease Eur Heart J Acute Cardiovasc Care. 2012;1:60-74.

13. Yusuf S, Zhao F, Mehta SR, et al. Effects of clopidogrel in addition to aspirin in patients with acute coronary syndromes without ST-segment elevation. N Engl J Med 2001;345:494-502.

14. Chen ZM, Jiang LX, Chen YP, et al. Addition of clopidogrel to aspirin in 45,852 patients with acute myocardial infarction: randomised placebo-controlled trial. Lancet 2005;366:1607-21.

15. Sabatine MS, Cannon CP, Gibson CM, et al. Addition of clopidogrel to aspirin and fibrinolytic therapy for myocardial infarction with ST-segment elevation. N Engl J Med 2005;352:1179-89.

16. Wallentin L, Becker RC, Budaj A, et al. Ticagrelor versus clopidogrel in patients with acute coronary syndromes. N Engl J Med 2009; 361:1045-57.

17. Wiviott SD, Braunwald E, Angiolillo DJ, et al. Prasugrel versus clopidogrel in patients with acute coronary syndromes. N Engl J Med 2007;357:2001-15.

18. Levine GN, Bates ER, Bittl JA, et al. 2016 ACC/ AHA guideline focused update on duration of dual antiplatelet therapy in patients with coronary artery disease: a report of the American College of Cardiology/American Heart Association Task Force on Clinical Practice Guidelines. J Am Coll Cardiol 2016;68: 1082-1115.

19. Valgimigli M, Bueno H, Byrne RA, et al. 2017 ESC focused update on dual antiplatelet therapy in coronary artery disease developed in collaboration with EACTS. Eur Heart J 2018;39:213-60.

20. Mauri L, Kereiakes DJ, Yeh RW, et al.; DAPT Study Investigators. Twelve or 30 months of dual antiplatelet therapy after drug-eluting stents. N Engl J Med 2014;371:2155-66.

21. Bonaca MP, Bhatt DL, Cohen M, et al. Long-term use of ticagrelor in patients with prior myocardial infarction. $\mathrm{N}$ Engl $\mathrm{J}$ Med 2015;372:1791-1800.

22. Navarese EP, Andreotti F, Schulze V, et al. Optimal duration of dual antiplatelet therapy after percutaneous coronary intervention with drug eluting stents: meta-analysis of randomised controlled trials. BMJ 2015;350:1618-8.

23. Palmerini T, Benedetto U, Bacchi-Reggiani L, et al. Mortality in patients treated with extended duration dual antiplatelet therapy after drug-eluting stent implantation: a pairwise and Bayesian network meta-analysis of randomised trials. Lancet 2015;385:2371-82.

24. Udell JA, Bonaca MP, Collet JP, et al. Long-term dual antiplatelet therapy for secondary prevention of cardiovascular 
events in the subgroup of patients with previous myocardial infarction: a collaborative meta-analysis of randomized trials. Eur Heart J 2016;37:390-9.

25. Rapsomaniki E, Thuresson M, Yang E, et al. Using big data from health records from four countries to evaluate chronic disease outcomes: a study in 114364 survivors of myocardial infarction. Eur Heart J Qual Care Clin Outcomes 2016;2:172-83.

26. Stone SG, Serrao GW, Mehran R, et al. Incidence, predictors, and implications of reinfarction after primary percutaneous coronary intervention in ST-segment-elevation myocardial infarction: the Harmonizing Outcomes with Revascularization and Stents in Acute Myocardial Infarction Trial. Circ Cardiovasc Interv 2014;7:543-51.

27. Bonaca MP, Storey RF, Theroux P, et al. Efficacy and Safety of Ticagrelor Over Time in Patients With Prior MI in PEGASUSTIMI 54. J Am Coll Cardiol 2017;70:1368-75.

28. Yusuf S, Mehta SR, Chrolavicius S, et al; Fifth organization to assess strategies in acute ischemic syndromes investigators. Comparison of fondaparinux and enoxaparin in acute coronary syndromes. N Engl J Med 2006;354:1464-76.

29. Stone GW, Witzenbichler B, Guagliumi G, et al. Bivalirudin during primary PCI in acute myocardial infarction. N Engl J Med 2008;358:2218-30.

30. Rao SV, O'Grady K, Pieper KS, et al. A comparison of the clinical impact of bleeding measured by two different classifications among patients with acute coronary syndromes. J Am Coll Cardiol 2006;47:809-16.

31. Budaj A, Eikelboom JW, Mehta SR, et al. Improving clinical outcomes by reducing bleeding in patients with non-ST-elevation acute coronary syndromes. Eur Heart J 2009;30:655-61.

32. Steg PG, Huber K, Andreotti F, et al. Bleeding in acute coronary syndromes and percutaneous coronary interventions: position paper by the working group on thrombosis of the european society of cardiology. Eur Heart J 2011;32:1854-64.

33. Costa F, van Klaveren D, James S, et al. Derivation and validation of the predicting bleeding complications in patients undergoing stent implantation and subsequent dual antiplatelet therapy (PRECISE-DAPT) score: a pooled analysis of individualpatient datasets from clinical trials. Lancet 2017;389:1025-34.

34. Cosentino N, Campodonico J, Faggiano P, et al. A new score based on the PEGASUS-TIMI 54 criteria for risk stratification of patients with acute myocardial infarction. Int $\mathrm{J}$ Cardiol 2019;278:1-6.

35. Bonaca MP, Bhatt DL, Oude Ophuis T, et al. Long-term tolerability of ticagrelor for the secondary prevention of major adverse cardiovascular events: A secondary analysis of the PEGASUS-TIMI 54 Trial. JAMA Cardiol 2016;1:425-32.

36. Bonaca MP, Bhatt DL, Steg PG, et al. Ischaemic risk and efficacy of ticagrelor in relation to time from P2Y12 inhibitor withdrawal in patients with prior myocardial infarction: insights from PEGASUS-TIMI 54. Eur Heart J 2016;37:1133-42.

37. Bhatt DL, Fox KA, Hacke W, et al. Clopidogrel and aspirin versus aspirin alone for the prevention of atherothrombotic events. N Engl J Med 2006;354:1706-17.

38. Yeh RW, Kereiakes DJ, Steg PG, et al. Benefits and risks of extended duration dual antiplatelet therapy after PCI in patients with and without acute myocardial infarction. J Am Coll Cardiol 2015;65:2211-21.

39. Stone GW, Maehara A, Lansky AJ, et al. A prospective naturalhistory study of coronary atherosclerosis. N Engl J Med 2011;364:226-35.

40. D'Ascenzo F, Biondi-Zoccai G, Moretti C, et al. TIMI, GRACE and alternative risk scores in Acute Coronary Syndromes: a meta-analysis of 40 derivation studies on 216,552 patients and of 42 validation studies on 31,625 patients. Contemp Clin Trials 2012;33:507-14.

41. Bohula EA, Bonaca MP, Braunwald E, et al. Atherothrombotic risk stratification and the efficacy and safety of vorapaxar in patients with stable ischemic heart disease and previous myocardial infarction. Circulation 2016;134:304-13.

42. Pocock SJ, Huo Y, Van de Werf F, et al. Predicting two-year mortality from discharge after acute coronary syndrome: An internationally-based risk score. Eur Heart J Acute Cardiovasc Care 2017. doi: 10.1177/2048872617719638.

43. Eagle KA, Lim MJ, Dabbous OH, et al. A validated prediction model for all forms of acute coronary syndrome: estimating the risk of 6-month post-discharge death in an international registry. JAMA 2004;291:2727-2733.

44. Wang Y, Li J, Zheng X, Jiang Z, et al. Risk factors associated with major cardiovascular events 1 year after acute myocardial infarction. JAMA Network Open 2018;1:e181079.

45. Yeh RW, Secemsky EA, Kereiakes DJ, et al DAPT. Development and validation of a prediction rule for benefit and harm of dual antiplatelet therapy beyond 1 year after percutaneous coronary intervention. JAMA 2016;315:1735-49.

46. Baber U, Mehran R, Giustino G, et al. Coronary thrombosis and major bleeding after PCI with drug-eluting stents: risk scores from PARIS. J Am Coll Cardiol 2016;67:2224-34.

47. Ueda P, Jernberg T, James S, et al. External Validation of the DAPT Score in a Nationwide Population. J Am Coll Cardiol 2018;72:1069-78.

48. Parodi G, Bellandi B, Tarantini G, et al. Clinical events beyond one year after an acute coronary syndrome: insights from the RECLOSE 2-ACS study. EuroIntervention 2017;12:2018-24.

49. Bradley SM, Hess GP, Stewart P, et al. Implications of the PEGASUS-TIMI 54 trial for US clinical practice. Open Heart 2017;4:e000580.

50. Alexander JH, Lopes RD, James S, et al. Apixaban with antiplatelet therapy after acute coronary syndrome. N Engl J Med 2011;365:699-708.

51. Mega JL, Braunwald E, Wiviott SD, et al. Rivaroxaban in patients with a recent acute coronary syndrome. N Engl J Med 2012;366:9-19. 